\title{
Variable Condition Model and Scheduling Optimization of Heating System Based on Layered Water Tank
}

\author{
Qimin Wang (D), Wanzhuo Li $\mathbb{D}$, and Kaixin Song $(\mathbb{D}$ \\ School of Energy and Power, Shenyang Institute of Engineering, Shenyang 110136, China \\ Correspondence should be addressed to Qimin Wang; wangqm03@163.com
}

Received 3 December 2021; Accepted 10 January 2022; Published 22 February 2022

Academic Editor: Rui Wang

Copyright ( 2022 Qimin Wang et al. This is an open access article distributed under the Creative Commons Attribution License, which permits unrestricted use, distribution, and reproduction in any medium, provided the original work is properly cited.

Under the background of carbon peaking and carbon neutralization proposed by China, the development of energy storage technology is imperative. The heat consumption of building central heating is large, which can be used as an important means of load side energy storage. The mathematical model of the central heating system is established, the optimal simulation of the regenerative temperature stratified water tank in the heating system is carried out, and the optimal structure of the temperature stratified water tank is obtained. The heating power of the fully mixed water tank is compared with the indoor maintenance temperature, and the power consumption of the primary pump is reduced by $8.5 \%$. The energy utilization rate of the heating system is high and has high energy-saving potential.

\section{Introduction}

With the global climate change problem becoming increasingly prominent, peak carbon dioxide emissions and carbon neutrality have become hot topics all over the world. The annual greenhouse gas statistics report published on the website of the US Environmental Protection Agency is based on national energy use, agricultural data, and other statistical data and summarizes the greenhouse gases produced by all anthropogenic emission sources in the United States, thus forming the Catalogue of Greenhouse Gas Emissions and Carbon Sinks. The catalogue shows that the peak of carbon dioxide emissions in the history of the United States appeared in 2007, when the total amount of greenhouse gases emitted by the United States was equivalent to 7.25 billion tons of carbon dioxide. Like the United States, the peak of total greenhouse gas emissions in Japan also appeared in 2007, with the total emissions equivalent to 1.365 billion tons of carbon dioxide. The "analysis of greenhouse gas emission data" shows that the peak of EU greenhouse gas emission appeared in 1990, and its total emission was equivalent to 5.57 billion tons of carbon dioxide. The emission peaks of three main components, carbon dioxide, methane, and nitrous oxide, all appeared in 1990, and then the emission decreased year by year. However, because of the great differences in economic and social development among the 28 member countries of the European Union, the peak times of greenhouse gas emissions among the member countries appear first and then. The peak emission of greenhouse gases in 9 member countries, such as Germany and Bulgaria, appeared in 1990, while the peak emission of other member countries appeared from 1991 to 2008, among which Cyprus and Slovenia did not appear until 2008 [1].

In 2020, President Xi Jinping made a commitment to achieve peak carbon dioxide emissions by 2030 and carbon neutrality by 2060 in the general debate of the 75th United Nations General Assembly, which provided a starting point for forcing high-quality economic development, and also demonstrated the responsibility of big countries, which helped boost global confidence in coping with climate change. As the world's largest energy consumer and carbon emitter, achieving the goal of carbon neutrality puts forward higher requirements for China's energy and economic sustainable development. The goal of "double carbon" is leading and systematic to China's green and low-carbon development, which can bring multiple effects of 
environmental quality improvement and industrial development. Focusing on reducing carbon emissions is conducive to promoting the green transformation of economic structure, accelerating the formation of green production methods, and boosting high-quality development. Highlighting the reduction of carbon emissions is conducive to the coordinated treatment of traditional pollutants and greenhouse gas emissions and makes the improvement of environmental quality and the control of greenhouse gases have a significant synergistic effect. Emphasizing that reducing carbon emissions is everyone's responsibility is conducive to promoting the formation of a green and simple lifestyle, reducing the consumption and waste of material products, and realizing energy conservation and pollution reduction. Accelerating the pace of carbon emissions is conducive to guiding green technology innovation, accelerating the development of green and low-carbon industries, forming new growth points in the fields of renewable energy, green manufacturing, and carbon capture and utilization, and improving the global competitiveness of industries and economies. The challenge of "double carbon goal" is very arduous. The road to zero carbon in the first-developing countries is still quite long, and there is no shortcut in the second-developing countries. In recent years, although the growth rate of China's energy consumption has slowed down and carbon emissions have gradually entered a plateau, the proportion of fossil energy in China's energy consumption structure is still as high as $80 \%$. On the road of realizing double carbon, China needs to make continuous efforts in the fields of power, industry, construction, agriculture, etc., to reduce carbon emissions, plan the optimal strategic path, and strive to achieve the parallel of carbon emission reduction and economic development $[2,3]$.

Under the background of carbon neutrality, domestic renewable energy generation will become the main power source in the future, and energy storage will undoubtedly play an important role in it. The output of new energy has the characteristics of fluctuation and intermittence, which is significantly different from the change trend of electricity load, and it is difficult to predict the power. Because of the lack of corresponding market return mechanism, the proportion of flexible power supply in power system is low. Affected by factors such as coal-fired power capacity regulation and power grid security constraints, the utilization rate of some transregional transmission channels is not high. The peak characteristic of China's power load curve is obvious, and the duration of $5 \%$ peak load does not exceed 48 hours [4]. A series of factors make it more difficult to balance power in real time, which affects the consumption of new energy power. Under the background of large-scale development of new energy, how to solve the problems and challenges in grid-connected consumption is the key factor to achieve the goal of "double carbon." Energy storage technology is an important technical means to effectively relieve the pressure of large-scale renewable energy grid connection, which can not only smooth the intermittent renewable energy power generation output but also improve the frequency modulation and peak shaving capability of thermal power units, so it has been widely valued [5].
Under the guidance of peak carbon dioxide emissions and carbon neutral strategy, the energy storage industry is bound to usher in a more rapid development. However, at present, there are still some unfavorable factors in energy storage at the power supply side, such as the difficulty in breaking through new material technology, the imperfect energy storage business model, and the reduction of new energy subsidies. At present, technologies such as energy storage on the power supply side are mainly limited by the research and development of new materials. It is difficult for new energy storage materials to make breakthroughs in energy density, unit cost, and application safety. Side energy storage technology is immature, and several energy storage technologies are still in the demonstration application stage, without extensive application. At present, many energy storage projects on the power supply side do not take into account the wide-area self-smoothing characteristics of renewable energy, which leads to the unreasonable distribution position of energy storage projects and excessive capacity allocation [6].

When the system faces the above challenges, the sourceload cooperative energy storage system has great development potential. Energy storage of the source-load cooperative system is helpful to the development of renewable energy and alleviate the problems of energy depletion and environmental pollution. At the same time, it can avoid the shortcomings of uncertain power generation of renewable energy, give full play to the advantages of environmental protection and economy of renewable energy, and reduce the adverse effects after grid connection. As the main body, the energy storage of the source-load cooperative system participates in the electric energy trading in the electricity market, which can reasonably conduct the electric energy trading according to the level of electricity price and user load and reasonably select the electric energy trading time and quantity according to the peak-valley difference of electricity price. The difference between the power generated by renewable energy and the system load is partly traded with the large power grid, which can reduce wind and light abandonment and improve the economy of the system. It plays an important role in building a clean, low-carbon, safe, and efficient modern energy system and has social practical significance. Therefore, the source-charge cooperative energy storage system is an important research direction with social and economic value [7].

Nowadays, all countries in the world put the energy issue in a strategic position that concerns the national economy and people's livelihood. As the largest developing country in the world, China's rapid economic development is accompanied by a large amount of energy consumption. Among all energy consumption, building energy consumption accounts for a large proportion. According to statistics, it was built in China.

Building energy consumption accounts for about $30 \%$ of the total social energy consumption. Among all kinds of building energy consumption in China, central heating energy consumption accounts for about $36 \%$ of the total national building energy consumption, which is the largest component of building energy consumption [8]. Therefore, 
load side energy storage is particularly prominent. The load energy storage system has the advantages of peak shaving and valley filling, demand response, and so on. Reasonable allocation of energy storage system is helpful to stabilize load fluctuation, reduce peak-valley difference of power grid, and increase precise control means. It is an effective measure to solve many problems caused by new energy grid connection, promote new energy consumption, and improve system operation reliability [9]. Our government has also promoted a series of related policies to encourage the optimal scheduling of energy storage at the user side. The "13th FiveYear Plan" clearly indicates that the future energy storage market will be extended to public utilities, from transmission and distribution side to load side [10]. Under this background, the energy storage system on the load side has great potential, and the development of energy storage on the load side has become an irreversible trend.

\section{Materials and Methods}

\subsection{Establishment of Dynamic Model of Heating System}

2.1.1. Introduction to Dynamic Models of Heating Systems at Home and Abroad. In applied energy in 1993, Andersson carried out simulation calculation according to the mathematical model of pipe network heat storage. In the adjustment, the heat storage of pipe network was considered and the heat storage capacity of heating pipe network was not considered. The operation parameters of the heating system were found to be more reasonable, and the purpose of energy saving was achieved [11]. Bohm and others did dynamic modeling of heating system and optimization of district heating in 1995. In this paper, a dynamic simulation model was established according to the temperature and flow nodes of the heating network and the district heating parameters were optimized [12].

In 1995, Bennonysson et al., in the Journal of Data Transfer Unit, put forward that according to the structure of the heating network, a dynamic model was established according to the temperature and flow of each node of the heating network for simulation, so that the change of the pipeline flow and the change of the temperature in the pipeline were obtained when the pipeline layout was known [13]. Bojic and Trifunovic published a work in Building and Environment in 2000, and a simple central heating pipe network was studied by establishing the corresponding mathematical equations by using the method of steady-state backstepping and continuous linear programming. This pipe network includes a heat source, a primary pipe network, a heat exchange station, a secondary pipe network, and three heat users, and the central heating pipe network was optimized [14]. Sauer et al. published in Applied Ergonomics in 2008, in which they studied the central heating system, simulated the heating system by using a computer, input different parameters through its interface in the simulation system, and displayed the simulation results, as well as provided the users with a display of energy consumption and discussed the design of the central heating system [15]. In the long-term dynamic simulation of Flynn and O'Malley natural boilers, the main parameters of the boiler system, such as main steam pressure and main steam temperature, are very reasonable and effective [16]. In 2004, according to the mathematical model, a central heating system model was established by Zaheeruddin, and the model was simulated by MATLAB. In the simulation, various control strategies were tested and analyzed and the existing heating regulation control strategies were optimized [17].

Lianzhong established a dynamic model of district hot water heating by using the conservation of mass and energy. Through simulation analysis, the influences of water supplement, radiator area, solar radiation, and water supply temperature on system operating parameters and users were analyzed. In the simulation system, different control strategies such as fuel control and temperature were combined and six different combinations of control strategies were simulated. The simulation results show that the control strategy based on user side can achieve the goal of energy saving and indoor temperature stability [18]. Matteo et al. used the sensitivity analysis method to study the relationship between building volume, window-wall ratio, and heating energy consumption and established a model for simulation. The error between the simulation result and the actual one was about 8\% [19]. Krzysztof et al. discussed the influence of outdoor temperature, solar radiation, and wind speed on heating heat load. Outdoor temperature directly affects the heat load of the heating system, and solar radiation can reduce the heat load of the heating system. With the increase of wind speed, the heat dissipation coefficient of the external surface of the building is improved, and at the same time, the cold air infiltration of the building is increased, so that the heat demand of the building is increased [20]. Jonsson et al. used the grey box method to simulate the heat load of central heating system, realized online prediction of heat load based on weather forecast data and SCADA system in central heating system, and established a model which can predict the heat load of regional central heating system [21]. Gabrielaitiene et al. realized computer simulation based on the physical model of a few nodes in district heating system and studied the temperature dynamics of district heating [22]. Peng et al., in Computer Simulation in 2002, studied the computer simulation of heating system, established the simulation model through building, heating network, and other modules, and introduced the solution ideas of the model [23].

In 2012, Chen and others simulated the heating system, taking the simple direct connection central heating system as an example, and analyzed the changes of indoor temperature when the outdoor temperature and water supply flow changed according to the model. As a result, the temperature response of the heating system was delayed, which provided a basis for on-demand heating [24]. In 2014, Xie and others designed a Smith-Fuzzy-PID controller for the established model and the model after the system parameters changed due to disturbance and other external factors and conducted simulation research. From the simulation results, the SmithFuzzy-PID controller is suitable for the case of interference between accurate input and external factors, which makes the system achieve better control effect [25]. 
In 2013, Xu et al. took the water supply flow and outdoor temperature as the input and the backwater temperature as the output of the simulated heating system and designed the fuzzy controller of the heating system. The fuzzy controller was simulated by using Fuzzy Toolbox, and the result can reach the expectation [26]. In 2008, Liu analyzed the running state of the heating system, carried out hydraulic calculation, and established a dynamic mathematical model. Using MATLAB/Simulink as a platform, she established a hydraulic system model of the heating pipe network and simulated the model. In the model, she could observe and test the hydraulic adjustment of the heating system and achieve the purpose of saving energy [27]. In his dissertation, Dang took the supercharged boiler unit as the research object, took Simulink as the simulation system, as the development platform, established the mathematical model of thermal equipment under certain simplified conditions, built the overall dynamic simulation model of the supercharged boiler, and analyzed the working principle of the boiler [28].

In his dissertation, Jin built a central heating simulation program with Simulink as the simulation system platform and then applied it to the built simulation program with BP neural network tool, which did not provide a basis for centralized adjustment [29].

2.1.2. Heating System Modeling in This Paper. In this paper, the heating system is decomposed into different subsystems and the mathematical model of each subsystem is established by using the centralized parameter method and heat balance equation, and the modules are combined into a complete simulation model of central heating system. In the model, different key parameters such as flow rate and load change curve can be obtained according to the temperature of supply and return water, different external environment, and indoor temperature. In this paper, parameters such as actual external air temperature are input into the model and the obtained system parameters are compared with the operation parameters of adding temperature stratified water tank, which proves the influence of temperature stratified hot water tank on system power and indoor temperature [30].

\subsubsection{Design Heat Load of Heating System}

(1) Design Heat Load of Heating System. It refers to the heat $Q$ supplied by the heating system to the building in unit time to reach the required indoor design temperature $t_{n}$ at a certain outdoor design temperature $t_{w}$ [31].

$$
Q=Q_{1 j}+Q_{1 x}+Q_{2}+Q_{3} \text {, }
$$

where $Q_{1 j}$ is the basic heat consumption of enclosure structure, $Q_{1 x}$ is the additional heat consumption of maintenance structure, $Q_{2}$ is the heat consumption of cold air penetrating into the room through the gap between doors and windows, which is called the heat consumption of cold air infiltration, and $Q_{3}$ is the heat consumption for heating cold air intruded by doors, holes, and adjacent rooms, which is called cold air intrusion heat consumption.

(2) Heat Consumption of Envelope. The basic heat consumption of envelope is as follows:

$$
Q_{1 j}=\mathrm{KF}\left(t_{n}-t_{w}\right) \alpha,
$$

where $K$ is the heat transfer coefficient of envelope, $F$ is the area of enclosure structure, $t_{n}$ is the calculated temperature in the heating room, $t_{w}$ is the outdoor calculated temperature of heating, and $\alpha$ is the temperature difference correction coefficient of envelope. The modified temperature difference factor is based on Table 1.

Heat transfer coefficient $k$ value of envelope:

$$
\begin{aligned}
K & =\frac{1}{R_{0}} \\
& =\frac{1}{R_{n}+R_{j}+R_{w}} \\
& =\alpha_{n}+\sum \frac{\alpha_{\lambda} \lambda}{\delta}+\alpha_{w},
\end{aligned}
$$

where $R_{0}$ is the heat transfer resistance of maintenance structure, $\alpha_{n}$ and $\alpha_{w}$ are the heat transfer coefficient of inner and outer surfaces of maintenance structure, $R_{n}$ and $R_{w}$ are the heat transfer resistance of internal and external surfaces of maintenance structure, $\delta$ is the thickness of each layer of the maintenance structure, $\lambda$ is the thermal conductivity of materials of each layer of the maintenance structure, and $R_{j}$ is the thermal resistance of individual material layers of maintenance structure composed of single or multilayer materials.

Calculation formula of additional heat consumption:

$$
\begin{aligned}
Q_{1} & =Q_{1 j}+Q_{1 x} \\
& =Q_{1 j}\left(1+\beta_{c h}+\beta_{f}+\beta_{l i}+\beta_{c}+\beta_{m}\right)\left(1+\beta_{g}\right)\left(1+\beta_{j}\right),
\end{aligned}
$$

where $Q_{1}$ is considered as the additional heat consumption of a certain enclosure, $Q_{1 j}$ is the basic heat consumption of a certain enclosure, $\beta_{c h}$ is orientation correction, $\beta_{f}$ is additional wind power, $\beta_{m}$ is external door attachment, $\beta_{g}$ is additional door height, $\beta_{l i}$ is modification of exterior walls on both sides, $\beta_{c}$ is the area ratio of window to wall made a big correction, and $\beta_{j}$ is additional clearance.

(3) Heat Consumption of Cold Air Infiltration. Estimate the heat consumption of cold air infiltration in the room according to the number of air changes in the room. The calculation formula is as follows:

$$
Q_{2}=0.278 c_{p} \rho_{w} V\left(t_{n}-t_{w}\right)
$$

where 0.278 is the unit conversion factor, $1 \mathrm{kj} / \mathrm{h}=0.278 \mathrm{w}, \mathrm{V}$ is the total air quantity infiltrated into the room through the gaps of doors and windows, $\rho_{w}$ is the air density at calculated temperature outside heating room, $c_{p}$ is the constant 
TABle 1: Modified temperature difference factor.

\begin{tabular}{|c|c|c|}
\hline $\begin{array}{l}\text { Serial } \\
\text { number }\end{array}$ & Envelope characteristics & $\begin{array}{c}\text { Modified } \\
\text { temperature } \\
\text { difference factor }\end{array}$ \\
\hline 1 & Exterior walls, roofs, floors, floors communicating with the outdoors, etc. & 1.00 \\
\hline 2 & Stuffy roof and floor slab above nonheating basement connected with outdoor air & 0.90 \\
\hline 3 & Partition wall adjacent to unheated stairwell with external doors and windows (1-6 floors building) & 0.60 \\
\hline 4 & Partition wall adjacent to unheated stairwell with external doors and windows (7-30 floors building) & 0.50 \\
\hline 5 & When there is a window on the external wall of the floor above the heating basement & 0.75 \\
\hline 6 & $\begin{array}{l}\text { When the floor above the heating basement has no window on the } \\
\text { external wall and is located above the outdoor floor }\end{array}$ & 0.60 \\
\hline 7 & $\begin{array}{c}\text { When the floor above the heating basement has no window on the } \\
\text { external wall and is located below the outdoor floor }\end{array}$ & 0.40 \\
\hline 8 & Partition walls and seismic joint walls adjacent to nonheating rooms with external doors and windows & 0.70 \\
\hline 9 & Partition wall adjacent to nonheating room without external doors and windows & 0.40 \\
\hline 10 & Expansion joint wall and settlement joint wall & 0.30 \\
\hline
\end{tabular}

pressure specific heat capacity of air, $t_{n}$ is the calculated indoor heating temperature, and $t_{w}$ is the outdoor calculated temperature of heating.

Average heating load [32]:

$$
Q_{a v}^{h}=Q_{h} \frac{t_{i}-t_{a v}}{t_{i}-t_{o}},
$$

where $Q_{a v}^{h}$ is the average heating load, $Q_{h}$ is the heating design heat load, $t_{i}$ is the calculated indoor heating temperature, generally taken as $18^{\circ} \mathrm{C}, t_{a v}$ is the average outdoor temperature of heating, and $t_{o}$ is the outdoor design temperature for heating.

According to the basic formula of heat calculation of heat exchanger, the heat transfer equation and heat balance equation are derived as follows [33]:

$$
\begin{aligned}
Q & =k A \Delta t_{m}, \\
Q & =q_{1} c_{1}\left(t_{1}^{\prime}-t_{1}^{\prime \prime}\right) \\
& =q_{2} c_{2}\left(t_{2}^{\prime \prime}-t_{2}^{\prime}\right),
\end{aligned}
$$

where $Q$ is the type-heat transfer, $k$ is the total heat transfer coefficient, $\Delta t_{m}$ is the average temperature difference of heat transfer, $q_{1}$ and $q_{2}$ are mass flow of inlet and outlet fluid, $c_{1}$ and $c_{2}$ are specific heat capacity of inlet and outlet fluids, and $t_{1}^{\prime}, t_{1}^{\prime \prime}, t_{2}^{\prime \prime}$, and $t_{2}^{\prime}$, respectively, represent the inlet and outlet temperatures of fluid on the primary side of heat exchanger and the inlet and outlet temperatures of heat exchanger on the secondary side.

2.1.4. Pipe Network Model. For the pipe network research, the lumped parameter method is also used to establish a mathematical model according to the heat balance. The heat balance of the heating pipe network mainly considers the heat dissipation loss and leakage loss of the pipeline. Because the water loss ratio is relatively small and it is impossible to know where to lose water, it is consistent to adopt the water loss rate per unit length of pipe section.

Therefore, in the simplified model, similar to a boiler, the heat brought by the pipe section minus the heat taken away and the heat absorbed by the supplementary cold water is the heat stored in the pipe section.
Calculation of heat dissipation per unit length of pipeline by the lumped parameter method: heat flowing into the pipeline is $c_{w} Q_{\text {in }}\left(T_{\text {in }}-T_{\text {out }}\right)$. Heat loss due to heat conduction in this section is $\left(T_{\text {out }}-T_{d b}\right) \times K$. Heat taken away by pipeline leakage is $a_{g b} b_{g b}\left(T_{\text {in }}-T_{\text {out }} / 2\right)$.

The heat transfer temperature of pipe section is calculated according to the average temperature of this pipe section, so the heat balance equation of pipe section is as follows [34]:

$$
C_{g d} \frac{\mathrm{d} T_{\text {out }}}{\mathrm{d} t}=c_{w} Q_{\text {in }}\left(T_{\text {in }}-T_{\text {out }}\right)-a_{\text {gl }} b_{\text {gl }} T_{\text {out }}-q_{\lambda},
$$

where $C_{g d}$ is the heat capacity of unit pipe section, $T_{\text {out }}$ is the outlet temperature of pipeline, $Q_{\text {in }}$ is flow into pipeline, $T_{\text {in }}$ is the temperature of inflow pipeline, $a_{g l}$ is the water leakage coefficient of pipeline, $0-1, b_{g l}$ is the water leakage of pipeline, and $q_{\lambda}$ is the heat dissipation loss of pipeline.

Among them, the pipe heat loss is described as follows: the water supply and return pipes are laid together in the heating pipe, but according to the practical experience and design requirements, the heat loss of the pipe network accounts for less of the transportation heat, so the additional thermal resistance between the common laying of the water supply and return pipes is not considered, and the calculation is directly made by a single pipe $q_{\lambda}$.

Heat loss of pipeline:

$$
\begin{aligned}
q_{\lambda} & =\frac{\left(T_{\text {out }}-t_{d b}\right)}{\sum R}, \\
R & =\frac{1}{2 \pi \lambda_{b}} \quad \ln \frac{d_{b}}{d_{g w}}+\frac{1}{2 \pi \lambda_{t}} \ln \frac{4 H}{d_{b}}, \\
H & =h+\frac{\lambda_{b}}{a_{k}}
\end{aligned}
$$

where $T_{\text {out }}$ is the outlet temperature of pipeline, $t_{d b}$ is the surface temperature, $\lambda_{b}$ is the thermal conductivity of insulation layer, $d_{b}$ is the outer diameter of insulation layer, $d_{g w}$ is the outer diameter of pipeline, $\lambda_{t}$ is the thermal conductivity of soil, $H$ is the converted depth of pipeline, $H$ is the depth from pipeline center to surface, and $a_{k}$ is the heat release coefficient of soil surface. 
2.1.5. Heat Exchanger Model. The main equipment of the heat exchange station is the heat exchanger, and it is particularly important to establish the mathematical model of the heat exchanger through reasonable mathematical methods. According to the heat transfer mechanism of the plate heat exchanger, because the heat capacity of the heat exchanger itself is small and the heat preservation measures are taken for the heat exchanger, the plate can be considered adiabatic and the heat loss can be ignored when establishing the mathematical model. According to the principle of heat balance, it can be concluded that the heat absorbed and released by cold and hot fluids is equal.

Referring to the nonlinear simulation method of establishing a simplified boiler model, the plate heat exchanger is considered as a lumped parameter system and the mathematical model is established by using the particle system method. Two inlet and outlet temperature nodes are established at the primary network side of the plate heat exchanger, and the temperature of the outlet temperature node represents the temperature of the hot fluid in the plate heat exchanger, so the heat entering the plate heat exchanger per unit time minus the heat transferred from the primary network side hot fluid to the secondary network side cold fluid is equal to the heat of the primary network side hot fluid temperature rise, and the mathematical equation is established as follows [35]:

$$
C_{\text {primary }} \frac{d T_{1 \text { out }}}{d t}=c_{w} G_{1}\left(d T_{1 \text { in }}-d T_{1 \text { out }}\right)-q_{j},
$$

where $C_{\text {primary }}$ is the water heat capacity of primary network side of plate heat exchanger, $d T_{1 \text { out }}$ is the outlet temperature of hot fluid at primary network side of plate heat exchanger, $T_{1 \text { in }}$ is the inlet temperature of hot fluid at primary network side of plate heat exchanger, $G_{1}$ is the flow rate of hot fluid at primary network side of plate heat exchanger, and $q_{j}$ is the heat exchange capacity of plate heat exchanger.

Similarly, for the secondary network side of the plate heat exchanger, two temperature nodes of inlet and outlet are also established and the temperature of the outlet temperature node represents the temperature of the cold fluid in the plate heat exchanger, so that the heat transfer amount of the plate heat exchanger minus the heat taken away by the secondary network side cold fluid through circulation is equal to the temperature rise of the cold fluid in the secondary network side of the heat exchanger, and the mathematical equation is established as follows:

$$
C_{\text {secondary }} \frac{\mathrm{d} T_{2 \text { out }}}{\mathrm{d} t}=q_{j}-c_{w} G_{2}\left(T_{2 \text { in }}-T_{\text {2out }}\right),
$$

where $C_{\text {secondary }}$ is the water heat capacity of primary network side of plate heat exchanger, $T_{2 \text { out }}$ is the outlet temperature of hot fluid at primary network side of plate heat exchanger, $T_{2 \text { in }}$ is the inlet temperature of hot fluid at primary network side of plate heat exchanger, and $G_{2}$ is the flow rate of hot fluid at primary network side of plate heat exchanger.
The formula for calculating the heat exchange capacity of the plate heat exchanger $q_{j}$ is

$$
q_{j}=\mathrm{KA} \Delta t_{p j}
$$

where $K$ is the heat transfer coefficient of plate heat exchanger, $A$ is the heat exchange area of plate heat exchanger, and $\Delta t_{p j}$ is the heat transfer temperature difference of primary and secondary network side of plate heat exchanger.

In the above formula, the heat transfer temperature is calculated by logarithmic mean temperature difference as follows:

$$
\Delta t_{p j}=\frac{\left(T_{1 \text { in }}-T_{2 \text { in }}\right)-\left(T_{1 \text { lout }}-T_{2 \text { out }}\right)}{\ln \left(T_{1 \text { in }}-T_{2 \text { in }} / T_{\text {lout }}-T_{\text {2out }}\right)} .
$$

The calculation formula of heat transfer coefficient $k$ of heat exchanger is

$$
K=\left(\frac{1}{\alpha_{1}}+\frac{1}{\alpha_{2}}+R_{w g}+R_{b p}\right)^{-1}
$$

where $\alpha_{1}$ is the convective heat transfer coefficient from heating medium to pipe wall, $\alpha_{2}$ is the convective heat transfer coefficient from pipe wall to heated medium, $R_{w g}$ is the water fouling thermal resistance, which is $1.16 \times 10^{-6} \mathrm{~m}^{2 \circ} \mathrm{C} / \mathrm{w}$ according to the Design Manual of Practical Heating and Air Conditioning, and $R_{b p}$ is the thermal resistance of plate, which is $4.5 \times 10^{-6} \mathrm{~m}^{2 \circ} \mathrm{C} / \mathrm{w}$ according to the Design Manual of Practical Heating and Air Conditioning.

2.1.6. Radiator Model. Taking the radiator as the lumped heat capacity model, the simplified model is the same as the pipe model, that is, the temperature change of the radiator is caused by the heat input minus the heat dissipation and the heat output. At the same time, in this study, users' radiator equipment in a divided building area is regarded as a whole and the temperature of supply and return water entering each user's radiator system is consistent under the condition of thermodynamic and hydraulic balance of each user's heating system, so this simplification is reasonable. The dynamic mathematical model of the radiator is established as follows when the inlet temperature of the radiator is $T_{g}$, the corresponding return temperature is $T_{h}$, and the indoor temperature is $t_{n}$ [36]:

$$
C_{s} \frac{d T_{h}}{d t}=c_{w} Q_{g}\left(T_{g}-T_{h}\right)-q_{s}
$$

The formula refers to the heat dissipation capacity of radiator. According to the heat dissipation capacity measurement method of heating radiator in GB13754-2017, the heat dissipation characteristic equation of radiator module is calculated $\left(q_{s}\right)$ :

$$
q_{s}=a_{s} F_{s}\left(\frac{T_{g}+T_{h}}{2}-t_{n}\right)^{1+b},
$$


where $\mathrm{C}_{s}$ is the heat capacity of radiator, $T_{h}$ is the return water temperature of radiator, $Q_{g}$ is the flow into radiator, $T_{g}$ is the radiator water supply temperature, $a_{s}$ is the radiator area coefficient, $0-1, F_{s}$ is the radiator area, and $1+b$ is the index of radiator standard characteristic formula.

Calculation of radiator area:

$$
F=\frac{Q}{K\left(t_{p j}-t_{n}\right)} \beta_{1} \beta_{2} \beta_{3} \beta_{4}
$$

where $F$ is the heat dissipation area, $K$ is the heat transfer coefficient of radiator, $t_{p j}$ is the average temperature of heat medium in radiator, $t_{n}$ is the calculated temperature in the heating room, $Q$ is the heat dissipation of the radiator, which is equal to the heat load of the heating room, $w, \beta_{1}$ is the correction factor of radiator assembly number, $\beta_{2}$ is the correction coefficient of radiator connection form, $\beta_{3}$ is the correction factor of radiator installation form, $\beta_{4}$ is the correction factor of flow into the radiator, and for $\beta_{1} \beta_{2} \beta_{3} \beta_{4}$, see "Practical Heating and Air Conditioning Design Manual" for the correction coefficient.

Simplified algorithm for quantity engineering of radiators:

$$
n=\frac{Q}{q}
$$

where $Q$ is the room heat load and $q$ is the heat dissipation capacity per unit number of radiators. Actual heat dissipation of radiator $=$ standard heat dissipation of radiator/ $\beta_{1} \beta_{2} \beta_{3} \beta_{4}$.

Hot water heating system:

$$
t_{p j}=\frac{\left(t_{s g}+t_{s h}\right)}{2}
$$

where $t_{s g}$ is the water inlet temperature of radiator and $t_{s h}$ is the outlet temperature of radiator.

Determination of inlet and outlet temperatures of radiators in each layer of single pipe system [36]:

$$
G_{L}=\frac{A \sum Q}{C\left(t_{g}-t_{s}\right)}
$$

2.1.7. Building Model. Because of the periodic disturbance of indoor and outdoor air temperature and sunlight, heat conduction is a complicated and unstable process. According to the theory of heat transfer, if the height and width of the wall are 8-10 times of the thickness, the calculation error is less than $1 \%$ when treated by one-dimensional heat conduction. Because the heat conduction along the height direction will not have a great influence on the indoor thermal environment, in order to simplify the calculation, a one-dimensional heat conduction model along the wall thickness direction is established in this study. The finite difference method is used to simulate, the inner surface of the wall is set as the origin of coordinates, the heat transfer problem is discretized in time and space, the difference equation is established, and the third boundary condition is considered, and the calculation is carried out layer by layer according to space.

In this study, there is no internal heat source in the wall of building envelope and the solar irradiance received by the exterior of the wall is included in the outer boundary point. At the same time, the relative humidity and absolute humidity in winter are very small. In order to simplify reasonably, the influence of moisture conduction and its phase change heat on the thermal physical parameters and temperature distribution of the wall is ignored. The heat conduction of the wall is a one-dimensional unsteady heat conduction process. The wall model is divided into three parts, namely, inner wall surface heat dissipation model, wall surface heat dissipation model, and outer wall surface heat dissipation model. The lumped parameter method is adopted in the wall heat dissipation models of the three parts.

The wall is divided into layers, the temperature of each layer is regarded as a point, and the thickness of each layer is subject to the subsequent calculation accuracy requirements. The model is divided into three parts, namely, the inner surface of the wall, the body of the wall, and the outer surface of the wall.

As shown in the figure, there is no heat storage on the inner surface and the inner surface is only a transformation from convective heat transfer to heat conduction, so the convective heat transfer on the inner wall surface is equal to the heat conduction from the wall surface to the inner wall. The schematic diagram of the wall structure is shown in Figure 1.

Heat dissipation calculation of inner wall surface [37]:

$$
h_{n}\left(t_{n}-T_{n q b}\right)=K_{q 1}\left(T_{n q b}-T_{q 1}\right) .
$$

Calculation of heat conduction in the wall:

The lumped parameter method is also used to conduct heat inside the wall. Because each divided level is the temperature of the current level, the division is about fine and the temperature in the wall is closer to reality. In the subsequent modeling of this paper, it will be divided according to the heat balance equation, so try to have more levels and divide the layers into $n$.

Temperature calculation of the first layer in the wall:

$$
\rho_{q 1} c_{q 1} \delta_{q 1} \frac{\mathrm{d} T_{q 1}}{\mathrm{~d} t}=K_{q 1}\left(T_{n q b}-T_{q 1}\right)-K_{q 2}\left(T_{q 1}-T_{q 2}\right) .
$$

Temperature calculation of the second layer in the wall:

$\rho_{q 2} c_{q 2} \delta_{q 2} \frac{\mathrm{d} T_{q 2}}{\mathrm{~d} t}=K_{q 2}\left(T_{q 1}-T_{q 2}\right)-K_{q 3}\left(T_{q 2}-T_{q 3}\right)$.

Temperature calculation of the $n$th layer in the wall, that is, where the wall is close to the outer surface:

$$
\rho_{q n} c_{q n} \delta_{q n} \frac{\mathrm{d} T_{q n}}{\mathrm{~d} t}=K_{q n}\left(T_{q n-1}-T_{q n}\right)-K_{q n}\left(T_{q n}-T_{w q b}\right) .
$$

Heat dissipation calculation of wall outer surface: like the inner surface, there is no heat storage on the outer surface, 


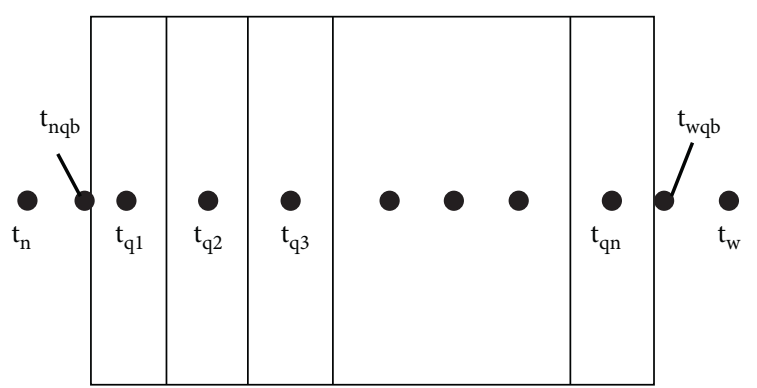

Figure 1: Schematic diagram of the wall.

but the outer surface only transforms from heat conduction to convection heat transfer. At the same time, the enclosure structure of the wall is still in contact with sunlight, so there is heat absorption, so the convection heat transfer on the outer wall surface is equal to the heat conduction from the wall to the outer surface.

$$
K_{q n} T_{q n}-T_{w q n}=h_{q w}\left(T_{w q b}-t_{w}\right)-q_{q s},
$$

where $h_{q n}$ is the convective heat dissipation coefficient between interior wall surface and indoor air, $t_{n}$ is the indoor air temperature, $T_{n q b}$ is the surface temperature of interior wall, $K_{q 1}$ is the heat transfer coefficient of the divided first wall, $T_{q 1}$ is the node temperature of the divided first floor wall, $\rho_{q 1}$ is the density of wall materials of the first layer divided, $c_{q 1}$ is the specific heat capacity of divided first layer wall materials, and $\delta_{q 1}$ is the thickness of the divided first wall, $\rho_{q n}, c_{q n}, \delta_{q n}, K_{q n}, T_{q n}$ are the density, specific heat capacity, thickness, heat transfer coefficient, and temperature of the divided $n$th wall, $T_{w q b}$ is the external wall surface temperature, $h_{q w}$ is the convective heat transfer coefficient between exterior wall surface and air, $t_{w}$ is the outdoor air temperature, and $q_{q s}$ is the heat intensity of external wall surface irradiated by the sun. Like the model of the roof and the wall, the dynamic mathematical model is the same as that of the wall.

In the living room, residents have furniture and various objects. As we all know, furniture itself is a kind of heat accumulator with heat storage characteristics. In this study, indoor furniture and various objects are regarded as objects $X$, and the objects $X$ are inserted into a certain insulation medium, so that only the leftmost end of the objects is exposed to the outside, and convection heat transfer is carried out on the surface of the objects $X$, as shown in Figure 2.

At the beginning, the equilibrium temperature of furniture is the initial indoor temperature for heating. When the indoor temperature changes, the leftmost end of the object will do surface flow heat exchange with the indoor temperature. In the model, the default is that the heat conduction in the object is extremely great and the temperature changes with time. Therefore, it is only necessary to consider the heat storage of the object itself, without considering the temperature changes in different internal coordinates, so it is not necessary to divide it into multiple layers like a wall. The dynamic heat transfer equation is

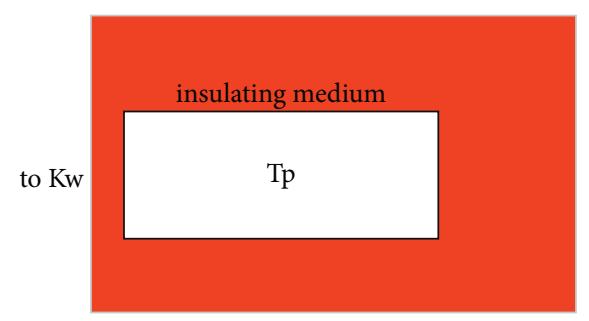

FIGURE 2: Schematic diagram of the regenerator model.

$$
C \frac{\mathrm{d} T_{\mathrm{p}}}{\mathrm{d} t}=K_{w}\left(t_{0}-T_{p}\right)
$$

where $C$ is the heat capacity of furniture and various objects, $T_{p}$ is the average temperature inside furniture, $K_{w}$ is the heat transfer coefficient of furniture, and $t_{0}$ is the indoor air temperature.

2.1.8. Hydraulic Model. The main tasks of hydraulic calculation of heating system pipeline are as follows:

(1) According to the flow rate of each pipe section of the known system and the circulating pressure (pressure head) of the system, determine the pipe diameter of each pipe section

(2) Determine the necessary circulating pressure (pressure head) of the system according to the known flow rate and pipe diameter of each section of the system

(3) According to the known pipe diameter of each pipe section of the system and the allowable pressure drop of this pipe section, determine the water flow through this pipe section

When designing a hot water heating system, in order to make the water flow of each pipe section in the system meet the design requirements and ensure that the water flow into each radiator meets the needs, it is necessary to carry out hydraulic calculation of the pipeline. When the fluid flows along the pipeline, energy will be lost due to the friction between the fluid molecules and the pipe wall. When the fluid flows through some accessories of the pipeline (such as valves, elbows, tees, and radiators), local vortices and impacts will occur due to the change of flow direction or speed and energy will also be lost. The former becomes the loss along the way, and the latter is called the local loss. Therefore, the resistance loss of a single calculated pipe section of the hot water heating system can be expressed by the following formula [38]:

$$
\Delta P=\Delta P_{y}+\Delta P_{j}=R l+\Delta P_{j},
$$

where $\Delta P$ is the total pressure loss, $\Delta P_{y}$ is the loss along the way, $\Delta P_{j}$ is the local loss, $R$ is the loss per unit length, specific friction, and $l$ is the length of pipe section. 
Among them, the specific friction calculation formula is

$$
\begin{aligned}
R & =\frac{\lambda}{d} \frac{\rho \nu^{2}}{2} \\
& =6.25 \times 10^{-8} \frac{\lambda}{\rho} \Delta \frac{G^{2}}{d^{5}},
\end{aligned}
$$

where $\lambda$ is the frictional resistance coefficient of pipeline, $d$ is the inner diameter of pipeline, $\rho$ is the density of heat medium, $G$ is the pipeline flow, and $v$ is the flow velocity of heat medium in the pipeline.

Hydraulic calculation methods used in this system: according to the changed flow of each pipe section of the most unfavorable circulation loop and the known pipe diameter of each pipe section, using the hydraulic calculation chart, determine the pressure loss of each pipe section of the circulating loop and the necessary circulating pressure of the system and check whether the head of the circulating pump meets the requirements.

The local loss of the pipe section can be calculated according to the following formula:

$$
\Delta P_{j}=\sum \xi \frac{\rho v^{2}}{2},
$$

where $\xi$ is the total local resistance coefficient in the pipe section.

The local resistance coefficient $\xi$ value of accessories (such as tee, elbow, and valve) of water flowing through hot water heating system can be consulted in the literature. The values given in the table are determined by experimental methods. With the above formula, the along-way loss $\Delta P_{j}$ and local loss $\Delta P_{j}$ of the spare pipe section in the system can be determined, respectively, and the sum of the two is the resistance loss of the pipe section.

The pump power is

$$
P_{g}=\frac{\rho g q_{v} H}{1000 \eta \eta_{m}},
$$

where $\rho$ is the density of fluid, $q_{v}$ is the volume flow, $H$ is the head, $\eta$ is the total efficiency of the pump, and $\eta_{m}$ is the efficiency of motor.

\section{Results and Discussion}

3.1. Difference between Fully Mixed Water Tank and Temperature Layered Water Tank. The traditional fully mixed heat storage tank has a simple structure, the high-temperature water supply pipe of the heat storage tank is located at the top of the heat storage tank, and the low-temperature return pipe is located at the bottom of the heat storage tank, so the supply and return water can be fully mixed in the heat storage tank, and the energy utilization efficiency is low. The structure of the traditional fully mixed water tank is shown in Figure 3. The cold water backwater from the client enters the heat storage tank from the left side of the tank, and the hot water flows out from the right side of the tank. For comparison, a water tank model of the same size with a middle baffle was established (Figure 4) to study the temperature transient temperature stratification of the new water tank and the ordinary water tank under the same boundary conditions.

3.2. Used Models and Parameters. Temperature stratified water tank is the energy stored in the central heating system and regulating equipment. By controlling the internal flow, reduce the mixing between high and low temperature water and realize the temperature stratification. Based on the influence of temperature on the heat transfer medium, density of buoyancy lift driver, which is formed by the water temperature from the bottom of the tank to the tank top, gradually increases with the formation of thermal stratification. For the influence factors of thermal stratification, the effect of Richardson's number $R i\left(R i=G r / R e^{2}\right)$ is mostly considered. Richardson number is the ratio or proportional dimensionless number of turbulent shear stress work and buoyancy work, and it is the criterion of atmospheric turbulence stability $[39,40]$.

Under the influence of gravity, the temperature stratification of the water tank is affected by the buoyancy force formed by the density gradient. $G r / R e^{2}$ is floating measure of the ratio of the inertial force of lift and flow; it is generally believed that $R i \leq 0.1$ is pure forced flow, and the research shows that when $R i \geq 3.6$, the tank inlet structure has an impact on temperature stratification and when $R i>10$, the influence of inlet on temperature stratification can be ignored or the influence of mixing on temperature stratification is very weak. For water tanks placed horizontally, $R i>0.2$, the stratification gradient in the water tank is very small, so the impact on the inlet of the water tank in turbulent ( $R i$ is small) and cannot be ignored. To realize temperature stratification in the water tank, it is necessary to consider the suppression of the turbulent flow regime. In order to meet the requirements of large water storage capacity and limited installation space of the water tank, and weaken the influence of the inlet effect of the water tank, the partition temperature stratified water tank studied in this paper can achieve better thermal zoning by adjusting the structure of different sections of the water tank and realize temperature stratification. As shown in Figures 3 and 4, the heat transfer medium (water) in the flow area inside the water tank was taken as the research object and a mathematical model was established. The length, width, and height of the water tank were $4 \mathrm{~m}, 2.5 \mathrm{~m}$, and $3 \mathrm{~m}$, respectively, and the inlet and outlet diameters were $100 \mathrm{~mm}$. The influence of the change of the baffle on the stratification under turbulence was studied, and the average temperature and temperature cloud map at the outlet of the water tank were analyzed, respectively. The center position of the round hole at the inlet and outlet of the water tank is shown in Figure 5.

3.3. Calculation of Control Volume. Because the heat storage model is complex in structure and difficult in calculation, some conditions that have little influence on calculation are 


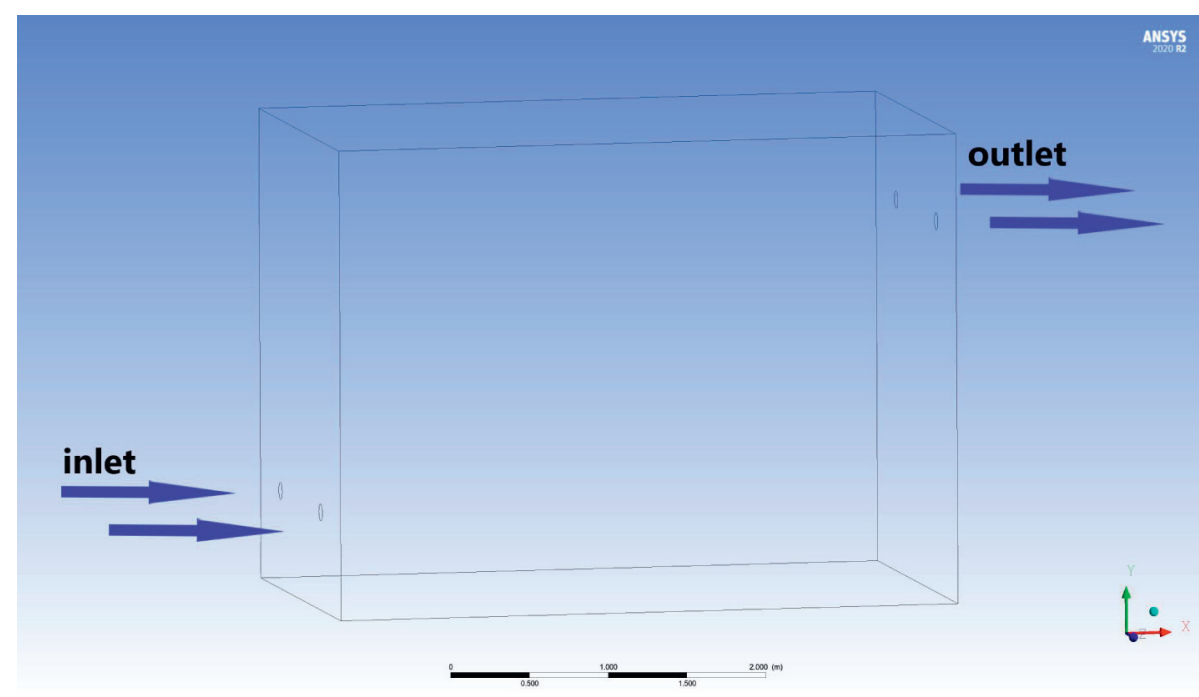

Figure 3: Physical model of the hot water tank.

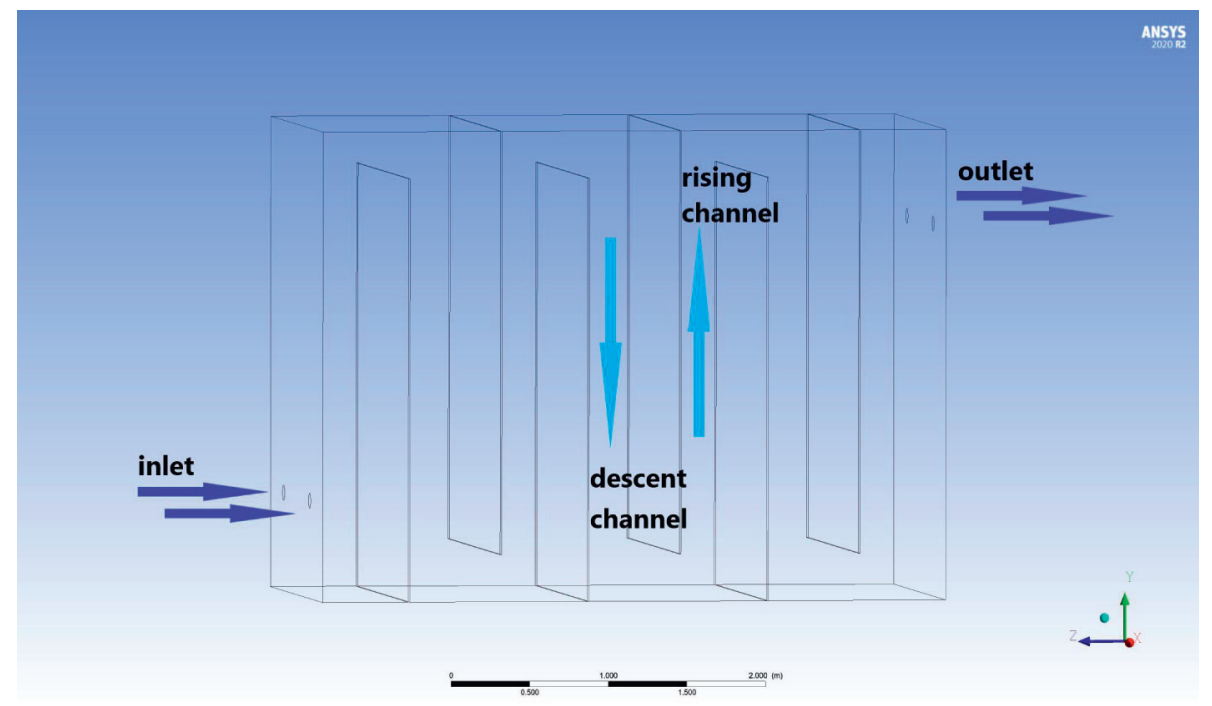

Figure 4: Schematic diagram of fluid flow direction.
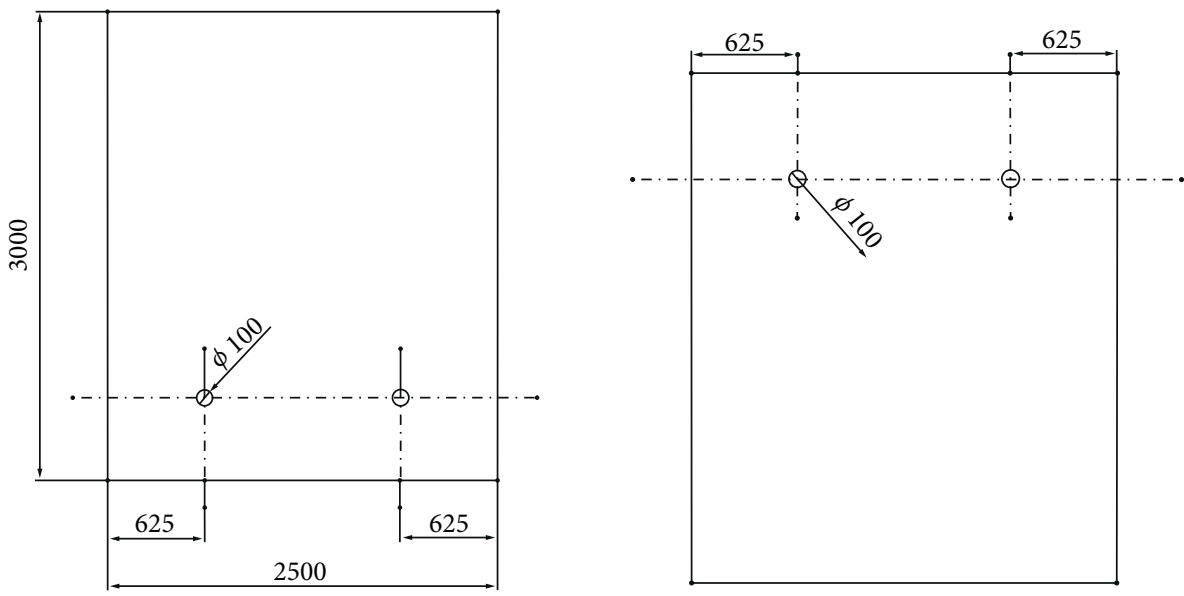

Figure 5: Layout of water tank inlet and outlet. 
approximated and ignored, and the following assumptions are given in combination with the relevant literature: the flow and heat transfer in the water tank are three-dimensional unsteady problems, and the governing equations for solving this problem are as follows.

Continuity equation [41]:

$$
\sum_{i=1}^{3} \frac{\partial}{\partial x_{i}}\left(\rho u_{i}\right)=0
$$

Momentum equation:

$$
\frac{\partial\left(\rho u_{i}\right)}{\partial t}+\sum_{j=1}^{3} \frac{\partial}{\partial x_{i}}\left(\rho u_{j} u_{i}\right)=-\frac{\partial P}{\partial x_{i}}+\sum_{j=1}^{3} \frac{\partial \tau_{i j}}{\partial x_{i}}+\rho f .
$$

Energy equation:

$$
\rho C_{p} \frac{\partial T}{\partial t}+\operatorname{div}(\lambda \overline{\operatorname{grad}} T)+T \beta \frac{\mathrm{d} P}{\mathrm{~d} t}+q+\phi .
$$

Among them,

$$
\begin{aligned}
\phi & =-\frac{2}{3} \eta(\operatorname{div} \bar{u})^{2}+2 \eta S_{i j} \frac{\partial u_{i}}{\partial x_{j}} S_{i j} \\
& =\frac{1}{2}\left(\frac{\partial u_{i}}{\partial x_{j}}+\frac{\partial u_{j}}{\partial x_{i}}\right) .
\end{aligned}
$$

Turbulence kinetic energy equation:

$$
\begin{aligned}
\rho \frac{\partial \varepsilon}{\partial t}+\rho u_{j} \frac{\partial k}{\partial x_{j}}= & \frac{\partial}{\partial x_{j}}\left[\left(\eta+\frac{\eta_{t}}{P r_{t}}\right) \frac{\partial k}{\partial x_{j}}\right] \\
& +\eta_{t} \frac{\partial u_{j}}{\partial x_{i}}\left(\frac{\partial u_{i}}{\partial x_{j}}+\frac{\partial u_{j}}{\partial x_{i}}\right)-C_{D} \rho \frac{k^{(3 / 2)}}{l_{m}} .
\end{aligned}
$$

Turbulence kinetic energy dissipation rate equation:

$$
\begin{gathered}
\left(\rho \frac{\partial \varepsilon}{\partial t}+\rho u_{j} \frac{\partial k}{\partial x_{k}}=\frac{\partial}{\partial x_{k}}\left[\left(\eta+\frac{\eta_{t}}{\sigma_{\varepsilon}}\right) \frac{\partial \varepsilon}{\partial x_{k}}\right]\right. \\
\left.+c_{1} \frac{\varepsilon}{k} \eta_{t} \frac{\partial u_{i}}{\partial x_{j}}\left(\frac{\partial u_{i}}{\partial x_{j}}+\frac{\partial u_{j}}{\partial x_{i}}\right)-C_{2} \rho \frac{\varepsilon^{2}}{k}\right) .
\end{gathered}
$$

The model and mesh processing are created by Geometry in ANSYS, and mesh optimization is imported. The boundary conditions are set and initialized in Fluent. The boundary condition of water tank inlet section is velocityinlet, and the velocity value is $0.038 \mathrm{~m} / \mathrm{s}$; the boundary condition of the outlet section of the water tank is set as outflow. Others are set as adiabatic boundary conditions. Cold water entering the water tank is always $50^{\circ} \mathrm{C}$, and the initial temperature in the water tank is $90^{\circ} \mathrm{C}$. Simulation calculation is done after setting iteration and control parameters. In order to ensure the accuracy of the calculation results, the grid has been verified to be irrelevant.

3.4. Structural Optimization. Considering the economy, it is best to place 6 separators in the water tank. The spacing of the separators in the water tank is optimized, and the fluid

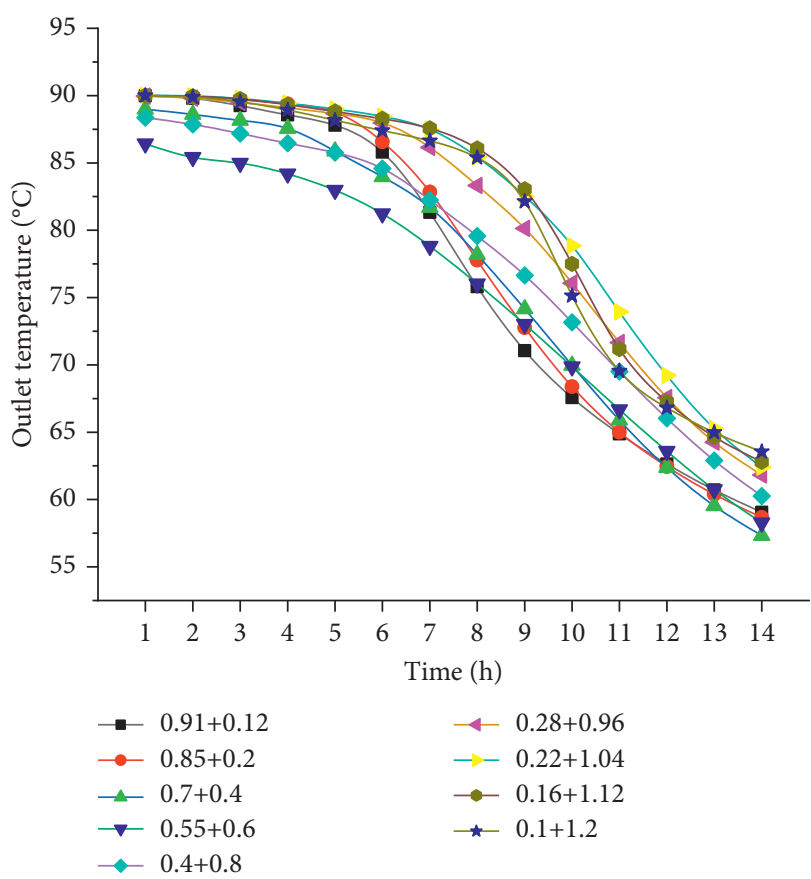

Figure 6: Influence of separator spacing on outlet temperature.

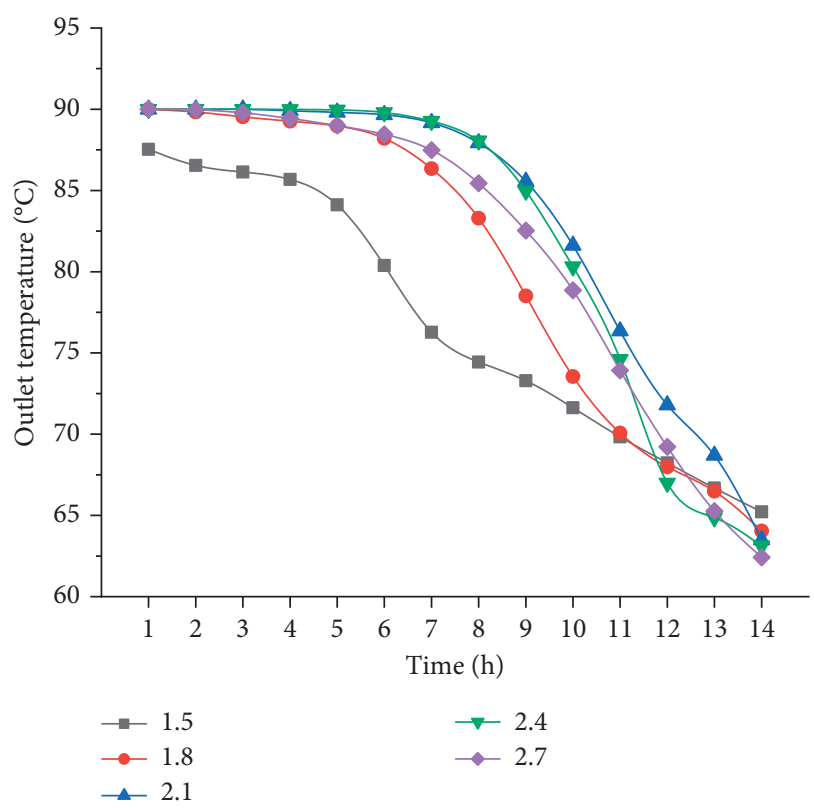

FIGURE 7: Influence of baffle height on average outlet temperature.

ascending channel and descending channel are $0.91+0.12$, $0.85+0.2, \quad 0.7+0.4, \quad 0.55+0.6, \quad 0.4+0.8, \quad 0.28+0.96$, $0.22+1.04,0.16+1.12$, and $0.1+1.2$, respectively. The partition height was $2.4 \mathrm{~m}$, and the outlet temperature at 14 hours was simulated (Figure 1). As can be seen from Figure 6, the average outlet temperature was relatively stable from 1 to 7 hours and the decline rate became faster after 7 hours. From the average outlet temperature at 14 hours, the best structure was $0.22 \mathrm{~m}$ for the ascending channel and $1.04 \mathrm{~m}$ for the descending channel. After that, the structure 


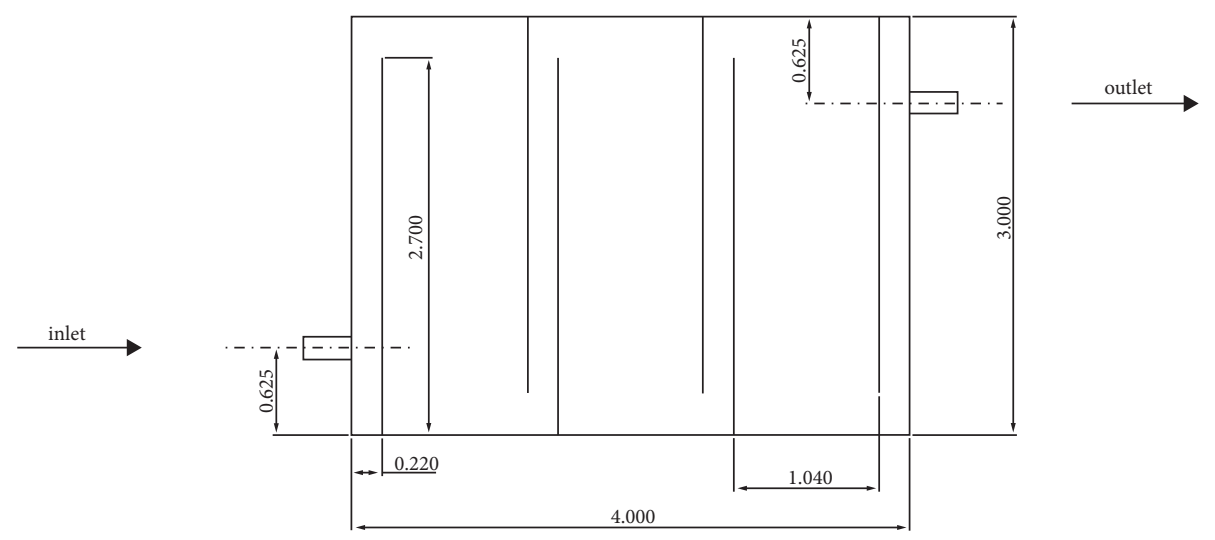

FIGURE 8: Structure diagram of the temperature layered water tank.

was optimized and the other conditions remained unchanged. The height of the partition was changed to $1.5 \mathrm{~m}$, $1.8 \mathrm{~m}, 2.1 \mathrm{~m}, 2.4 \mathrm{~m}$, and $2.7 \mathrm{~m}$, respectively. The 14-hour outlet temperature was simulated (Figure 7). It can be seen from Figure 2 that the mixing speed of hot and cold water is different with different partition heights, among which the best height is $2.7 \mathrm{~m}$.

3.5. Optimization Results. Based on the actual engineering, the research object is the temperature stratified heat storage tank in the clean energy heating system. The heat storage performance is simulated and analyzed, and the influence of each parameter on the outlet average temperature index is studied to provide reference for the optimization of operation parameters. On the basis of the research, the physical model and mathematical model of temperature stratified heat storage tank are established to study its heat storage performance, and the optimization analysis is carried out according to the numerical simulation results. Among them, the ascending passage is $0.22 \mathrm{~m}$, the descending passage is $1.04 \mathrm{~m}$, and the partition height is $2.7 \mathrm{~m}$, as shown in Figure 8. After optimization, compared with the 14-hour outlet temperature (Figure 9) of the fully mixed water tank, it is not difficult to find that the outlet temperature has been greatly improved.

\subsection{Influence of Fully Mixed Water Tank and Temperature} Stratified Water Tank on Optimal Operation of Central Heating System. The target building is the most common multistorey residence in hot summer and cold winter areas, with a total heating area of $1020 \mathrm{~m}^{2}$. According to the Design Code for Heating, Ventilation and Air Conditioning of Civil Buildings, the heating index of $70 \mathrm{~W} / \mathrm{m}^{2}$ is selected. The calculated outdoor temperature is $-16.9^{\circ} \mathrm{C}$, and the indoor design temperature is $18^{\circ} \mathrm{C}$. Take $30 \mathrm{~m}^{2}$ from the heat storage tank and plate heat exchanger parameters as shown in Table 2, from $7 \mathrm{pm}$ to $9 \mathrm{pm}$. Taking Table 3 as an example, the ratio of the pump power to the initial power for 14 hours in a day is calculated, and the result is shown in Figure 10.

As shown in Table 4, it can be seen that, from 7 am to $12 \mathrm{am}$, because of the temperature rise, the outlet water temperature did not significantly decrease, but the power

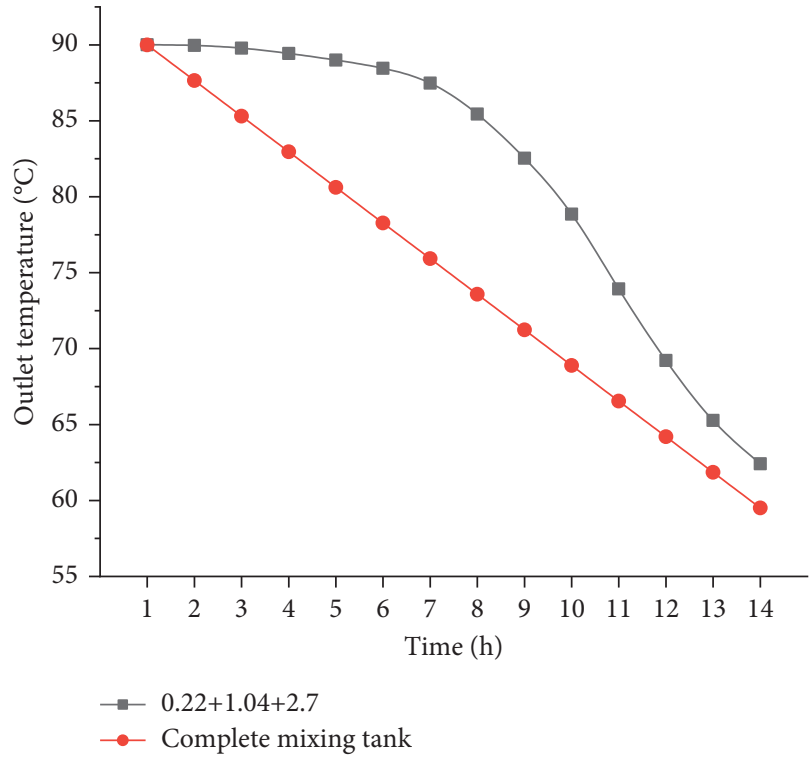

FIgURE 9: Comparison of average outlet temperature.

TABle 2: Parameters of the plate heat exchanger.

\begin{tabular}{lc}
\hline Total heat exchange area & $8 \mathrm{~m}^{2}$ \\
\hline Veneer heat exchange area & $0.385 \mathrm{~m}^{2}$ \\
Equivalent diameter of runner & $0.0072 \mathrm{~m}$ \\
Cross-sectional area of single runner & $0.001339 \mathrm{~m}^{2}$ \\
Plate thickness & $0.0008 \mathrm{~m}^{2}$ \\
Number of runner plates & 20 \\
\hline
\end{tabular}

ratio showed a downward trend, which was especially obvious in the thermal storage tank with temperature stratification.

From 12 o'clock to 4 o'clock in the afternoon, the outlet water temperature drops substantially and the indoor temperature in the completely mixed water tank system also drops accordingly. The temperature stratified water tank system can still maintain the original indoor design temperature, but it needs to increase the flow and increase the power of the pump. From 4 o'clock to 8 o'clock, the temperature stratified water tank system cannot maintain the 
TABLe 3: Temperature in a city on January 20, 2021.

\begin{tabular}{|c|c|c|c|c|c|c|c|c|c|c|c|c|c|c|}
\hline Time & 7 & 8 & 9 & 10 & 11 & 12 & 13 & 14 & 15 & 16 & 17 & 18 & 19 & 20 \\
\hline Temperature $\left({ }^{\circ} \mathrm{C}\right)$ & -22 & -21 & -19 & -17 & -14 & -12 & -11 & -9 & -9 & -9 & -9 & -10 & -11 & -11 \\
\hline
\end{tabular}

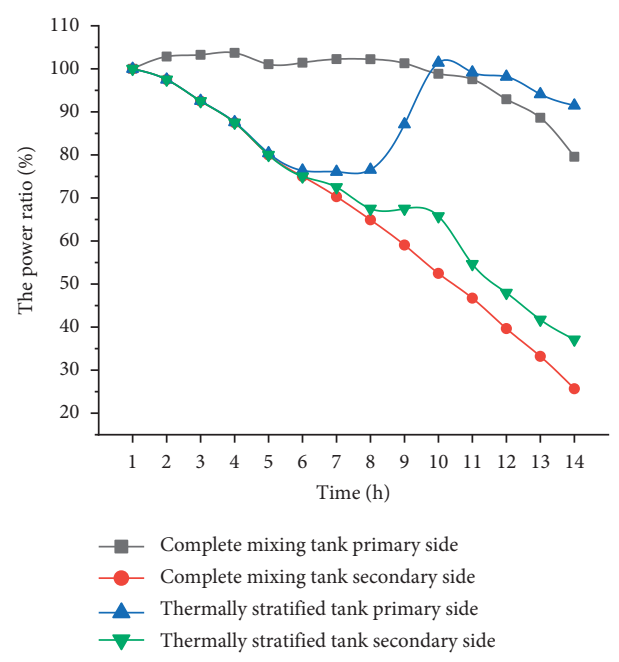

Figure 10: Power ratio of primary and secondary side pumps.

TABLE 4: Indoor temperature.

\begin{tabular}{|c|c|c|c|c|c|c|c|c|c|c|c|c|c|c|}
\hline Completely mixed $\left({ }^{\circ} \mathrm{C}\right)$ & 18 & 18 & 18 & 18 & 18 & 18 & 13.4 & 13.9 & 7.55 & 2.92 & 0.107 & -4.25 & -7.37 & -8.54 \\
\hline Separator $\left({ }^{\circ} \mathrm{C}\right)$ & 18 & 18 & 18 & 18 & 18 & 18 & 18 & 18 & 18 & 17.9 & 4.24 & -1.57 & -5.59 & -6.63 \\
\hline
\end{tabular}

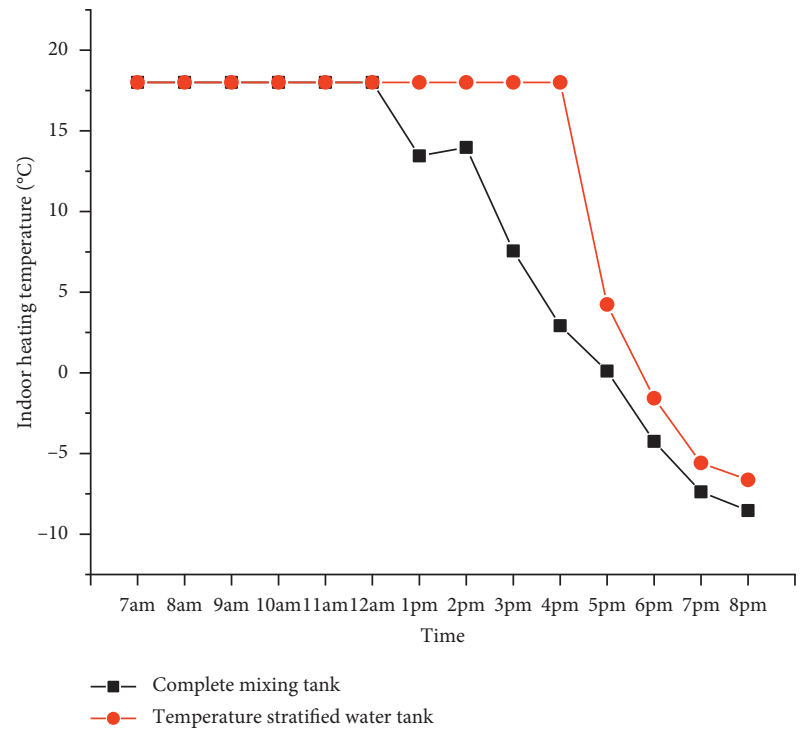

FIGURE 11: Indoor temperature change.

indoor temperature slide; at this time, the power ratio slowly declines. When the outlet temperature drops to a certain extent, the heat transfer cannot maintain the indoor temperature. At this time, the indoor temperature can be calculated by the heat transfer. The comparison results between the fully mixed water tank and the temperature stratified water tank are shown in Figure 11. It can be concluded that the temperature stratified water tank is obviously helpful to the maintenance of indoor temperature in the heating system.

As shown in Table 4, by observing the indoor temperature of the central heating system with fully mixed water tank and temperature stratified water tank, it can be concluded that the application effect of temperature stratified water tank heat storage is better. As the heat storage system takes part in the peak regulation of thermal power plants and as the heat storage system combines with wind power, photoelectric power, and nuclear power to replace conventional energy to realize district heating alone, the heat storage system is getting bigger and bigger. The volume of a single heat storage tank can even reach hundreds of thousands of cubic meters. In this case, the thermal storage mode of temperature stratified water tank is bound to achieve greater economic benefits.

\section{Conclusions}

In this paper, the model of the heat storage tank of the central heating system is established, the overall structure of the heat storage tank is designed, and a new heat storage tank structure is obtained to improve the efficiency of the heating system. The partition arrangement and partition height of temperature layered thermal storage tank are simulated and calculated, and the recommended arrangement and height are obtained. On the basis of the previous simulation results, the power and indoor temperature of central heating are calculated. Finally, by comparing the power and temperature 
of heating system under two different storage modes of hot water tank, the following results are obtained:

(1) A heat storage water tank structure which can better realize the layered operation of the heat storage water tank is obtained. On the $4 \mathrm{~m} \times 2.5 \mathrm{~m} \times 3 \mathrm{~m}$ water tank, 6 baffles are placed so that the ascending channel is $0.22 \mathrm{~m}$, the descending channel is $1.04 \mathrm{~m}$, and the baffle height is $2.7 \mathrm{~m}$.

(2) The layout and height of the partition in the temperature layered thermal storage tank are simulated, which improves the average outlet temperature.

(3) Through a specific project, the 14-hour power ratio and indoor temperature of the two kinds of thermal storage tanks are compared and calculated without changing other conditions, which proves the economy of the temperature stratified water tank.

\section{Data Availability}

The datasets generated for this study are available on request to the corresponding author.

\section{Conflicts of Interest}

The authors declare that the research was conducted in the absence of any commercial or financial relationships that could be construed as potential conflicts of interest.

\section{Acknowledgments}

The authors acknowledge the teacher Wang Qimin for his guidance and assistance.

\section{References}

[1] G. Liu, "The United States, Japan and Europe have reached the peak of carbon emissions many years ago: dajiang Geba turns around," Environment and Life, vol. 21, pp. 16-20, 2014.

[2] J. Xi, "Carrying forward the past and forging ahead into the future, and starting a new journey of global response to climate change," People's daily, vol. 2, 2020.

[3] J. Su, Y. Liang, L. Ding, G. Zhang, and L. He, "Discussion on China's energy development strategy under the goal of carbon neutrality," Journal of Chinese Academy of Sciences, vol. 9, pp. 1001-1009, 2021.

[4] K. Chongqing and L. Yao, "Key scientific issues and theoretical research framework of high-proportion renewable energy power system," Power System Automation, vol. 9, pp. 2-11, 2017.

[5] J. Young, R. Xia, and W. Yang, "Peak carbon dioxide emissions, research on the development trend of new energy under the background of carbon neutralization," China Engineering Consulting, vol. 9, pp. 22-26, 2021.

[6] J. Tong, Q. Hong, H. Lu, R. Wu, and Y. Guangyao, "Overview of the development status and application prospect of energy storage technology on the power supply side," Huadian Technology, vol. 7, pp. 17-23, 2021.

[7] M. Gao, Planning of Small and Medium-Sized Source-LoadStorage Cooperative System Based on Electricity Market, Northeast Dianli University, Jilin, China, 2021.
[8] J. Jin, “Analysis of China's building energy consumption trends and energy conservation priorities," Green building materials, vol. 11, p. 34+37, 2019.

[9] Li Yue, Research on Optimal Power Load Dispatching Based on Energy Storage System at User Side, Zhejiang University, Hangzhou, China, 2018.

[10] National Development and Reform Commission, "The 13th five-year plan for renewable energy development," Shanghai Building Materials, vol. 1, p. 78, 2017.

[11] S. Andersson, "Influence of the net structure and operating strategy on the heat load of a district-heating network," Applied Energy, vol. 46, no. 2, pp. 171-179, 1993.

[12] B. Bohm and H. F. Ravn, "Operational ptimization in a district heating system," Energy Conversion and Management, vol. 36, pp. 297-314, 1995.

[13] A. Benonysson, Dynamic Modeling and Operational Optimization of District Heating Systems. Denmark, pp. 10-15, Technical Uni. of Denmark, Kongens Lyngby, Denmark, 1995.

[14] M. Bojic and N. Trifunovic, "Linear programming optimization of heat distribution in a district-heating system by valve adjustments and substation retrofit," Building and Environment, vol. 35, no. 2, pp. 151-159, 2000.

[15] J. Sauer, D. G. Wastell, and C. Schmeink, "Designing for the home: a comparative study of support aids for central heating systems," Applied Ergonomics, vol. 40, no. 2, pp. 165-174, 2009.

[16] M. E. Flynn and M. J. O’Malley, "A drum boiler model for long term power system dynamic simulation," IEEE Transactions on Power Systems, vol. 14, no. 1, pp. 209-217, 1999.

[17] L. Li and M. Zaheeruddin, "A control strategy for energy optimal operation of a district heating," International Journal of Energy Research, vol. 28, no. 7, pp. 597-612, 2004.

[18] L. Li, "Simulation of characteristics and control strategy of indirect connection district hot water heating system," Energy Saving, vol. 9, pp. 8-15, 2008.

[19] M. Caldera, S. P. Corgnati, and M. Filippi, "Energy demand for space heating through a statistical approach: application to residential buildings," Energy and Buildings, vol. 40, no. 10, pp. 1972-1983, 2008.

[20] K. Wojdyga, "An influence of weather conditions on heat demand in district heating systems," Energy and Buildings, vol. 40, no. 11, pp. 2009-2014, 2008.

[21] G. R. Jónsson, "A model for predicting the yearly load in district heating systems," Proceedings of the Institution of Mechanical Engineers - Part A: Journal of Power and Energy, vol. 216, no. 3, pp. 277-281, 2005.

[22] I. Gabrielaitiene, B. Bøhm, and B. Sunden, "Modelling temperature dynamics of a district heating system in Naestved, Denmark-A case study," Energy Conversion and Management, vol. 48, no. 1, pp. 78-86, 2007.

[23] L. Peng, B. Yu, and X. Tu, "Computer simulation design and implementation of large heating system," Computer Simulation, vol. 4, pp. 56-58, 2002.

[24] L. Chen, Y. Song, and Y. Zhang, "Simulink simulation and analysis of central heating system," Energy Saving, vol. 12, pp. 22-23, 2012.

[25] Y. Xie, Y. Yang, and M. Xie, "Research on the application of Smith-Fuzzy-PID in central heating control system," Computer Measurement and Control, vol. 22, pp. 2823-2825, 2014.

[26] . Tong, G. Cui, and Y. Qu, "MATLAB simulation of fuzzy control central heating controller," District Heating, vol. 1, pp. 39-42, 2013. 
[27] H. Liu, "Research on energy saving of MATLAB simulation in district heating pipe network," Industrial Architecture, vol. 38, pp. 128-132, 2008.

[28] Z. Dang, Modeling and Dynamic Characteristics of Boiler Based on Matlab/Simulink, Master Thesis, Master Thesis of North China Electric Power University, Beijing, China, 2014.

[29] C. Jin, Study on Dynamic Model of Heat Storage and Heat Load Prediction of central Heating System, North China Electric Power University, Beijing, China, 2017.

[30] R. Wang, Q. Sun, P. Tu, J. Xiao, Y. Gui, and P. Wang, "Reduced-order aggregate model for large-scale converters with inhomogeneous initial conditions in DC microgrids," IEEE Transactions on Energy Conversion, vol. 36, no. 3, pp. 2473-2484, Sept. 2021.

[31] Y. Lu, Z. Ma, and P. Zou, HVAC, China Building Industry Press, Beijing, China, Third edition, 2015.

[32] S. Li and H. Kang, Manual of Central Heating, pp. 42-50, China Electric Power Press, Beijing, China, 1995.

[33] S. Yang and W. Tao, Heat Transferpp. 484-485, Higher Education Press, Beijing, China, Fourth edition, 2006.

[34] S. Wang, Simulation and Analysis of a Boiler Room Heating System, Beijing University of Technology, Beijing, China, 2018.

[35] S. Qian, Design Manual of Heat Exchanger, Chemical Industry Press, Beijing, China, 2002.

[36] L. Zongjiang, C. Liang, J. Li, and L. Changling Airong, "Comparative study on standard working conditions of thermal performance test of heating radiator," $H V A C$, vol. 46, no. 11, pp. 73-77, 2016.

[37] J. Zhang, Simplification of Thermal Model of Heating Buildings and Simulation of Accident Conditions, Harbin Institute of Technology, Harbin, China, 2018.

[38] C. He and L. Guo, Pumps and Fans, China Electric Power Publishing House, Beijing, China, Fourth edition, 2008.

[39] R. Wang, Q. Sun, W. Hu, Y. Li, D. Ma, and P. Wang, "SoC-based droop coefficients stability region analysis of the battery for stand-alone supply systems with constant power loads," IEEE Transactions on Power Electronics, vol. 36, no. 7, pp. 7866-7879, 2021.

[40] R. Wang, Q. Sun, D. Ma, and Z. Liu, “The small-signal stability analysis of the droop-controlled converter in electromagnetic timescale," IEEE Transactions on Sustainable Energy, vol. 10, no. 3, pp. 1459-1469, 2019.

[41] Y. Li, Study on the Dynamic Characteristics of Solar Energy Tank Energy Storage Based on CFD and its Thermodynamic Performance Optimization Analysis, Shandong Jianzhu University, Jinan, China, 2016. 